\title{
Manufacturing Process Monitoring in Terms of Energy Management Improving
}

\author{
A. Simo, C. Barbulescu, S. Kilyeni, C. Dragos
}

\author{
Attila Simo*, Constantin Barbulescu, Stefan Kilyeni \\ Power Systems Department \\ Politehnica University Timisoara, Romania \\ attila.simo@upt.ro, constantin.barbulescu@upt.ro, stefan.kilyeni@upt.ro \\ *Corresponding author: attila.simo@upt.ro
}

\section{Claudia Dragos}

Department of Automation and Applied Informatics

Politehnica University Timisoara, Romania

claudia.dragos@aut.upt.ro

\begin{abstract}
Seeking out new technologies and deploying them for competitive advantage is a key priority for any company. In this context, Internet of Things (IoT) promise to change the way we live and work. IoT could help us in overtaking the top global challenges, as energy management, agriculture and food security, environment and natural resources security, etc., but to achieve this vision, "things" need to sense their environment and share this information among them as well as with us to offer intelligent decision-making. Nowadays, IoT become even more interesting due to the existence of Low Power Wide Area (LPWA) networks. LPWA is a key component of a wider IoT technology wave. In this paper, a LoRaWAN based solution is presented, for manufacturing process monitoring, in order to improve energy management in plants. The authors present a minimally invasive device, with which many product manufacturing data can be obtained for analysis and further improvements. This paper is an extended variant of $[14]^{a}$.

Keywords: intelligent decision-making, LoRaWAN communication protocol, wireless sensor network.

\footnotetext{
${ }^{a}$ Partially reprinted and extended, with permission based on Licence Number 4587520206486 C) IEEE, from "2018 7th International Conference on Computers Communications and Control (ICCCC)."
}

\section{Introduction}

This paper presents a part of a more complex work from the Low Power Wide Area (LPWA) networks field, ongoing in the Power Systems Department from Politehnica University Timisoara, in collaboration with ETA2U Innovation.

The current paper is an extended version of [14]. It deals with energy management improvement, based on wireless sensor network and the LoRaWAN communication protocol, dedicated especially to factories with industrial buildings placed over a large geographical area $(6 \mathrm{~km}$ distance between buildings). In [14] it was presented a battery powered non-invasive device, isolated from any other equipment on the production lines, developed by ETA2U Innovation in collaboration with Politehnica University Timisoara. The job of this device was to collect the data about the manufacturing process, to transmit them over long distances using the LoRaWAN communication protocol. In this paper the new version of this device is presented. This new device brings some improvements, like communication via Ethernet and optocoupler for connections with programmable logic controllers.

LPWA represent a new communication paradigm, which will complement traditional cellular and short-range wireless technologies in addressing diverse requirements of IoT applications. 
LPWA technologies offer unique sets of features including wide-area connectivity for low power and low data rate devices, not provided by legacy wireless technologies [1]. These type of networks are unique because they make different tradeoffs than the traditional technologies prevalent in IoT landscape such as short-range wireless networks (Z-Wave, Zig-Bee) [13], legacy wireless local area networks (WLANs), and cellular networks (GSM, LTE), etc. [8].

The legacy non-cellular wireless technologies are not ideal to connect low power devices distributed over large geographical areas because the range of these technologies is limited to a few hundred meters at best. The devices, therefore, cannot be arbitrarily deployed or moved anywhere, which is a requirement for many applications for logistics, smart city and agriculture [17]. Legacy WLANs, on the other hand, are characterized by shorter coverage areas and higher power consumption for machine-type communication. The range of these technologies is extended using a dense deployment of devices and gateways connected using multihop mesh networking [4].

It is obvious, due to the advantages, the LPWA filed is a priority for the mobile industry, and initiatives have been ongoing for several years in an attempt to deliver standards that will enable mobile operators to offer LPWA-like connectivity. With a range of a few to tens of kilometers and battery life of ten years and be-yond, LPWA technologies are promising for the Internet of low-power, low-cost, and low throughput things [9]. A very long range of LPWA technologies enables devices to spread and move over large geographical areas, thus IoT and M2M devices connected by LPWA technologies can be turned on anywhere and anytime to sense and interact with their environment [5].

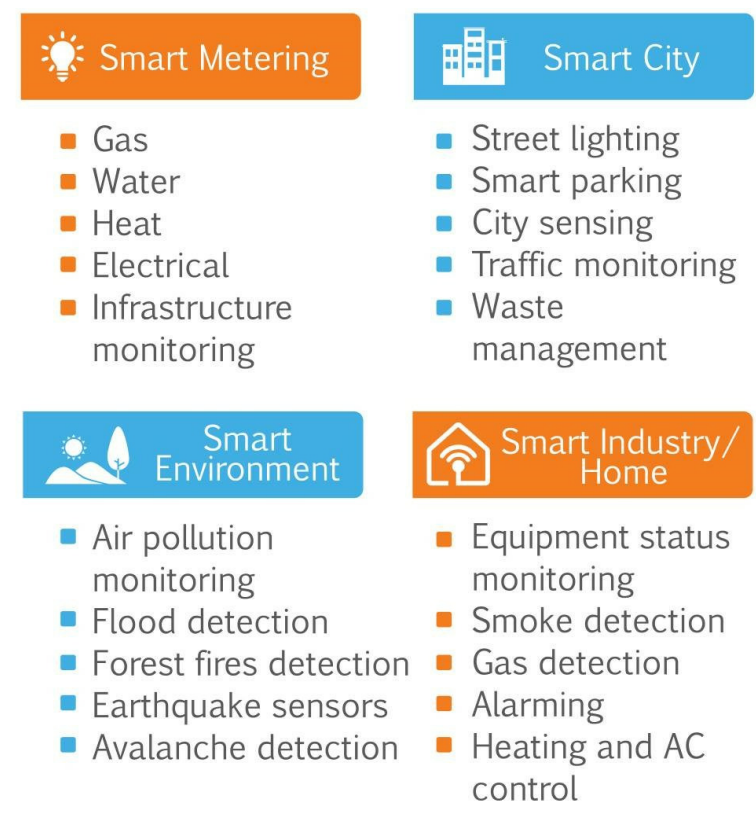

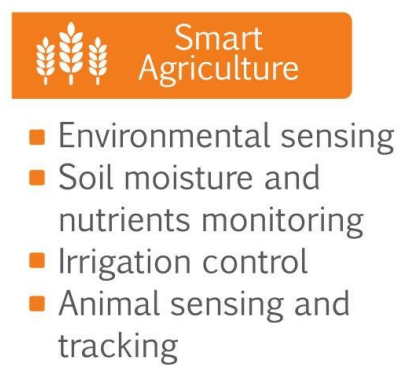

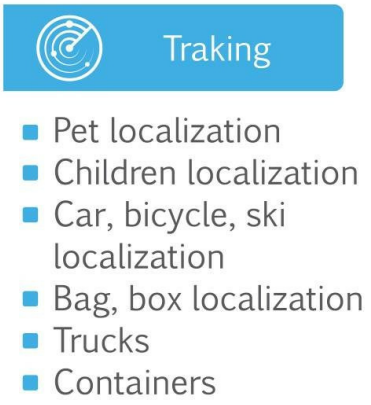

Figure 1: Applications of LPWA technologies across different sectors

Fig. 1 and Fig. 2 highlights the variety of applications across several business sectors that can exploit Low Power Wide Area technologies to connect their end devices. These sectors include smart city, smart grid, smart metering, logistics, industrial monitoring, agriculture, wildlife monitoring, environment monitoring, tracking etc.

It is worth clarifying that LPWA technologies achieve long range and low power operation at 

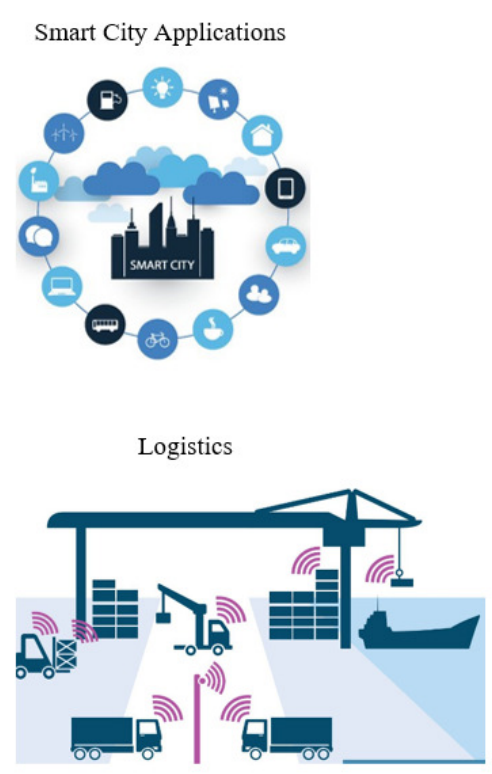
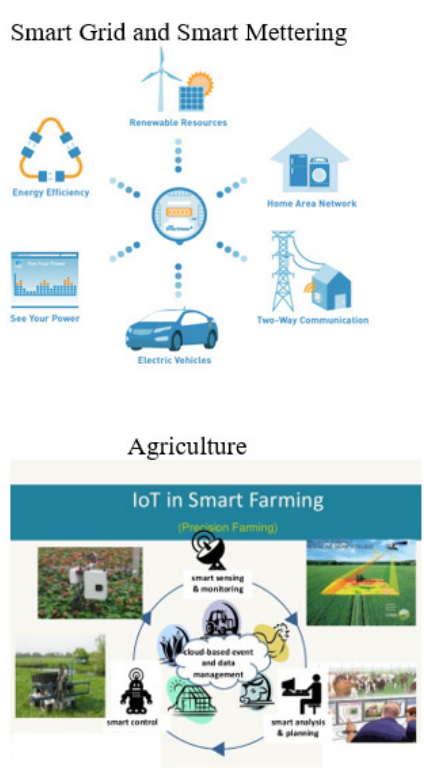
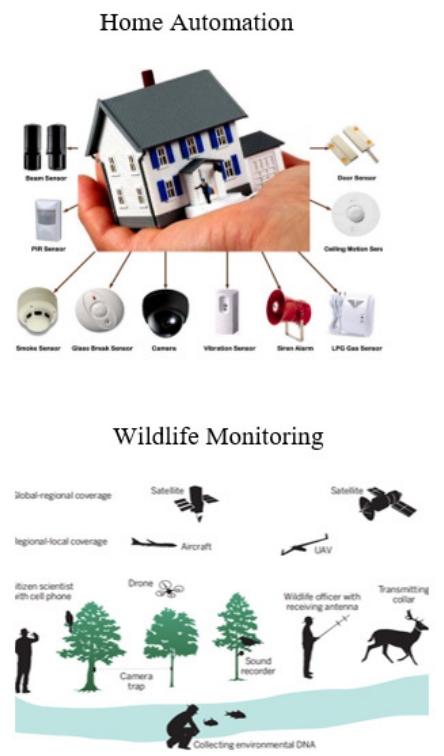

Figure 2: LPWA technologies in different sectors

the expense of low data rate (typically in orders of tens of kilobits per seconds) and higher latency (typically in orders of seconds or minutes). Therefore, it is clear that LPWA technologies are not meant to address each IoT use case. Specifically, LPWA technologies are considered for those use cases that are delay tolerant, do not need high data rates, and typically require low power consumption and low cost. The Internet of Things, as a new growth engine of the information and communications technology industry, has sparked global enthusiasm.

IoT promise to change the way we live and work. IoT could help us in exceeding the top global challenges due to energy crisis, resource depletion, environmental pollution, etc. To achieve this vision, "things" need to sense their environment, share this information among them as well as with us to offer intelligent decision making.

Nowadays IoT become even more interesting due to the existence of LPWA networks.

Different studies have forecasted a growth in volume and revenue of IoT in the next several years. Number of connected devices and consumer electronics will surpass the number of human subscribers using mobile phones, personal computers, laptops and tablets by 2020 [13]. Moving forward, by 2024, the overall IoT industry is expected to generate 4.3 trillion dollars' revenue across different sectors such as device manufacturing, connectivity, and other value added services [15]. Recent improvements in cheap sensor and actuation technologies along with an emergence of novel communication technologies are all positive indicators, supporting the forecasted trends [11].

Nowadays, energy efficiency in manufacturing processes provides several advantages to companies, such as cost saving notwithstanding rising and volatile energy prices. In [6] the authors define green products as those that have been manufactured while consuming as little energy as possible - not just products which consume less energy when used by the customer. Consequently, the best practices that aim to reduce energy consumption during manufacturing process are increasingly important to today's manufacturing companies.

In many companies, energy management practices at manufacturing process levels suffer due to lack of awareness of energy consumption behavior. In fact, energy savings are expected to be achievable both from improvements in energy efficiency of specific manufacturing processes, as well as from the usage of innovative energy monitoring systems and management approaches [16]. 
New emerging autonomous technologies, such as IoT, are enhancing monitoring of manufacturing processes. IoT technology (e.g. smart meters, sensors) provide awareness of energy consumption patterns by collecting real-time energy consumption data.

The availability of real-time energy consumption data gives us several opportunities to reduce energy consumption by enabling and enhancing energy-efficient practices in manufacturing process management [15].

The focus of this research is to build a more flexible device, as the one presented in [14], that helps us track manufacturing process in order to increase the efficiency of electricity consumption - e.g. identifying causes and reducing the downtimes between producing two elements.

In the 1st section generalities about LPWA are discussed, the 2nd one refers to the LoRa technology and the LoRaWAN communication protocol. In 3rd section the new monitoring device is presented, and the results and discussions are tackled. Finally, conclusions are synthesized within the 4 th section.

\section{The LoRa technology}

LoRa is a proprietary physical layer for LPWA connectivity. A physical layer technology that modulates the signals in SUB-GHz ISM band using a proprietary spread spectrum technique developed and commercialized by Semtech Corporation. A bidirectional communication is provided by a special chip spread spectrum technique, which spreads a narrow band input signal over a wider channel bandwidth. The resulting signal has noise like properties, making it harder to detect or jam.

A special interest group constituted by several commercial and industrial participants dubbed as $L o R a^{T} M$ Alliance proposed LoRaWAN, an open standard defining architecture and layers above the LoRa physical layer. A simple ALOHA scheme is used at the MAC layer that in combination with LoRa physical layer enables multiple devices to communicate at the same time but using different channels and/or orthogonal codes (spreading factors). End devices can hop on to any base station without extra signaling overhead. The base stations connect end devices via a backhaul to network server, the central unit of the LaRaWAN system that suppresses duplicate receptions, adapts radio access links, and forwards data to suitable application servers (fig. 3). Application servers then process the received data and perform user defined tasks.

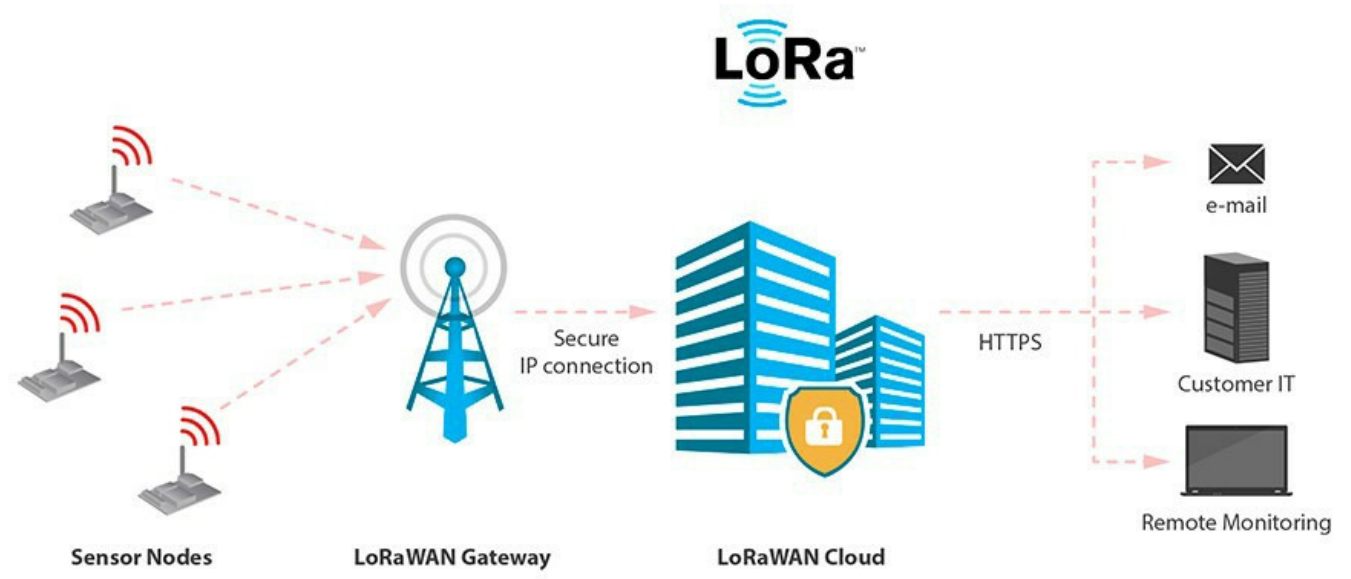

Figure 3: Architecture of a LoRa based application

LoRaWAN anticipates that the devices will have different capabilities as per application 
requirements. Therefore, LoRaWAN defines three different classes of end-devices, all of which support bidirectional communication but with different downlink latency and power requirements:

- Class A device achieves the longest lifetime but with the highest latency. It listens for a downlink communication only shortly after its uplink transmission.

- Class B device, in addition, can schedule downlink receptions from base station at certain time intervals. Thus, only at these agreed-on epochs, applications can send control messages to the end devices (for possibly performing an actuation function).

- Class C device is typically mains-powered, having capability to continuously listen and receive downlink transmissions with the shortest possible latency at any time.

LoRaWAN standard uses symmetric-key cryptography to authenticate end devices with the network and preserve the privacy of application data [13].

Several studies evaluated LoRaWAN in real world environments including outdoor and even indoor settings. For example, a study in [9] observed $15 \mathrm{~km}$ and $30 \mathrm{~km}$ communication ranges for LoRaWAN on ground and water respectively in Oulu Finland.

In another study [11], end devices transmitted at $14 \mathrm{dBm}$ using highest spreading factor to the base station that was located within $420 \mathrm{~m}$ radius. The packet delivery ratio at the base station is recorded to be $96.7 \%$.

In [10] the authors evaluated the performance of LoRaWAN unconfirmed uplink data frames in an indoor environment. They first showed the limitations in term of periodicity and size of data because of the ISM band regulation in a default channel configuration. Such regulation also limits the maximum amount of data that can be sent per day. Then, they evaluated the signal quality received from different locations, in order to verify the feasibility to cover an entire building with the LoRaWAN technology. The difference in the composition of walls between the rooms and the lab floors had not much impact on the quality of the transmission and packet loss. Only communications with the basement experienced degradations. They also showed that the data rate can be a factor of loss and should be selected appropriately when configuring end-device. Finally, they showed the average current consumption of a LoRa mote and how the used data rate can affect the global energy consumption. [7] propose an architecture of smart health monitoring system for smart factory and proposes the methodology for maximizing the productivity.

In [2], first part of the paper, the authors are dealing with electrical and heat energy multipoint measurements and consumption reduction options. They highlights current studies, obtained findings and first results, focusing on industrial and office type building electrical energy consumption, definition of problems and description of possible experimental solution. In [3], the second part, they present the first measurement data and analysis results and prove the need for a mathematical model and prediction algorithm development for improved and energy efficient building regulation processes. They conclude that LoRAWAN network is well suited for the non-dynamic sensor data transfer, but not for detailed electrical power consumption, thus a new approach for data aggregation is needed, by means of embedded algorithms already within the power measurement equipment.

The use of a long-range (LoRa) technology, originally developed for IoT, is investigated with the aim of implementing DMSs is presented in [12]. After the conclusion that LoRa and LoRaWAN architectures show a good match with measurement systems, this paper focuses on the characterization of time-related performance indicators that are important for distributed systems. The experimental results show the capability of low-cost transceiver to schedule the 
transmission of frames with a standard uncertainty less than $3 \mu$ s and an acceptable long-term clock stability (Allan Deviation) of commercial available devices (nodes and packet forwarders) for application such as smart metering, smart building, and process industry.

\section{Results and discussions}

In Romania, unfortunately in case of many plants, the industrial buildings are placed over large geographical area, few kilometers, in most cases these are not equipped with an Internet connection or other related facilities (due to the impact of the investments). Monitoring the manufacturing process using XXI century technologies is hampered by these disadvantages.

Decision-makers, process engineers are always looking to improve the manufacturing process. They want to have clear production statistics - how many equipment were produced on the line per hour, per shift handover, per day, seeking the human or machines efficiency. They are also interested about the downtime between the production of two equipment, in term of energy management. In several cases, as we received requests decision-makers want these data to be transmitted over long distances. In the absence of the Internet, these things are difficult to achieve.

The main purpose of [14] is to provide solution for some particular requirements from such plants. They need a monitoring device to increase energy management efficiency, which at the same time is able to transmit data over long distances, over the air.

In this direction, in the laboratories of ETA2U - IoT solutions integrator company - by ETA2U Innovation was developed and tested a monitoring device to meet the expectations of these factories.

Minimal intervention on production lines, low consumption of our equipment and long-range data transmission were the special requirements of customers [14].

The developed equipment consists of 3 main parts:

- Non-invasive Split Core Current Transformer, type YHDC 100A-STC-013-000 - which takes the parameters from one phase of the circuit.

- Arduino Mega (ATmega 1280 - Fig. 4) with a Dragino Lora Shield v1.4 (Fig. 5) - for reading, processing and transferring the collected data using LPWA technology.

- Own developed Printed Circuit Board (PCB) (fig. 13) - through which the connection to the technological process is made. Mainly this part of the device has been further developed. An Ethernet port and optocouplers have been added, the last ones for connection to the manufacturing process via programmable logic controllers.e.

This type of microcontroller has been chosen due to the energy efficiency and cost benefits. Also, it PowerSaving, SleepMode, SleepModeWatchDog features have played an important role in our decisions. It is user friendly and is easy to implement applications on it. Thanks to these functions, this microcontroller reaches a typical consumption of about $0.013 \mathrm{~mA}$, by which we partly responded to customer requirements.

One of the customer's special requirement it was that after the data has been collected, to be transmitted over large geographical area. We did not have the possibility of transmission the data via Internet. For this case, we have chosen to use LoRa technology (again taking into account that we need to have low energy consumption). We chose to add to our device the Dragino LoRa Schield v1.4. This device is a long-range Arduino transmitter emitter that works with an open source library. It allows the user to send and receive data at long distances with low transfer rate. 


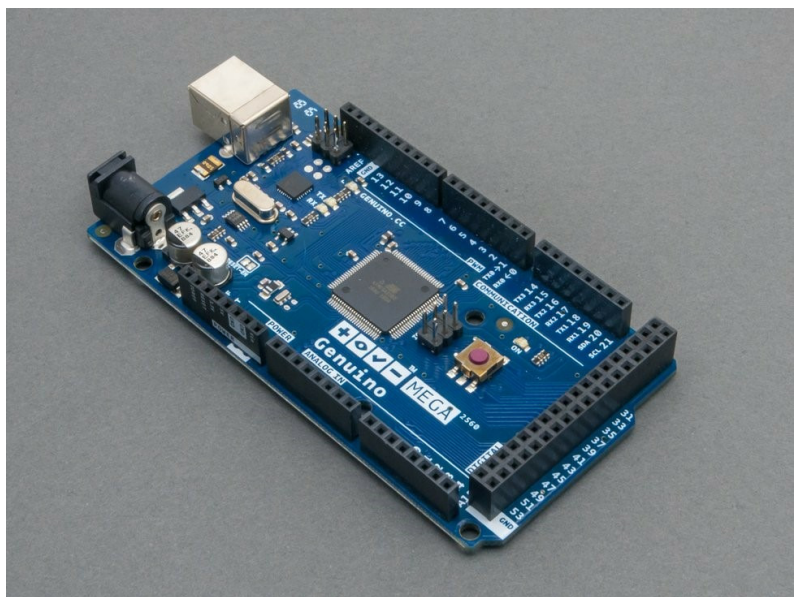

Figure 4: Arduino Mega - for reading, processing and transfer data

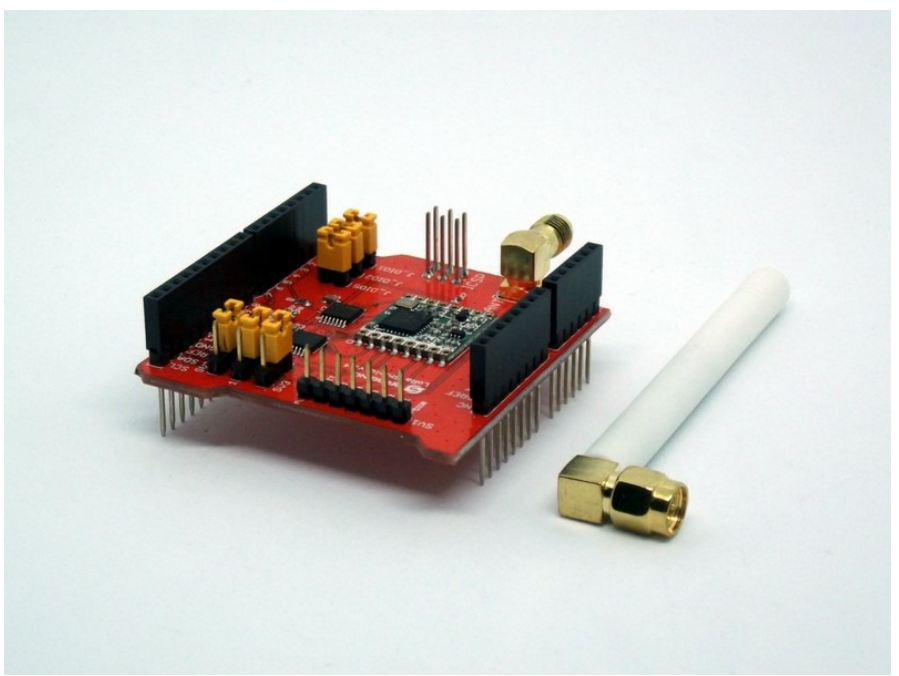

Figure 5: Dragino Lora Shield - long range data transmission

This shield (fig. 5) is based on a RFM98W radio frequency transmitter, which can be used with professional wireless sensor systems applications. Thanks to its high sensitivity up to $-148 \mathrm{dBm}$ combined with a $+20 \mathrm{dBm}$ power amplifier, it makes it the best choice for remote communication applications.

The PCB between the current transformer and the microcontroller has a double role:

- The first is to "wake up" the microcontroller - because when there is no activity on the production line the controller enters in standby mode. For this, we used an LM393 comparator to generate an interruption on Arduino Mega's number 21 digital pin. This is done by the voltage difference between the reference + and fixed - terminals. When a consumer voltage value is connected to the terminal - it will exceed the reference value at terminal + . This will generate an output signal that will "wake up" the microcontroller.

- The second one is to connect the current transformer sensor to the microcontroller. The output signal from the current transformer needs to be conditioned so it meets the input requirements of the controller analog inputs.

Fig. 6 shows the scheme of this intermediate circuit [14], which has the following components 
(each of them calculated, according to our needs):

- 3 x $10 \mathrm{kOhm}$ resistor;

- 1 x 97 Ohm resistor (burden resistance to close the circuit);

- 1 x $22 \mathrm{kOhm}$ resistor;

- 1 x $50 \mathrm{kOhm}$ resistor;

- $1 \times 10 \mu \mathrm{F}$ capacitor;

- $1 \times 1000 \mu \mathrm{F}$ capacitor;

- 1 x $27 \mu \mathrm{F}$ capacitor;

- 1 x LM393 comparator.

The circuit consists of five main parts:

- the current transformer sensor and the burden resistor;

- the comparator;

- the biasing voltage divider;

- Ethernet port;

- optocouplers.

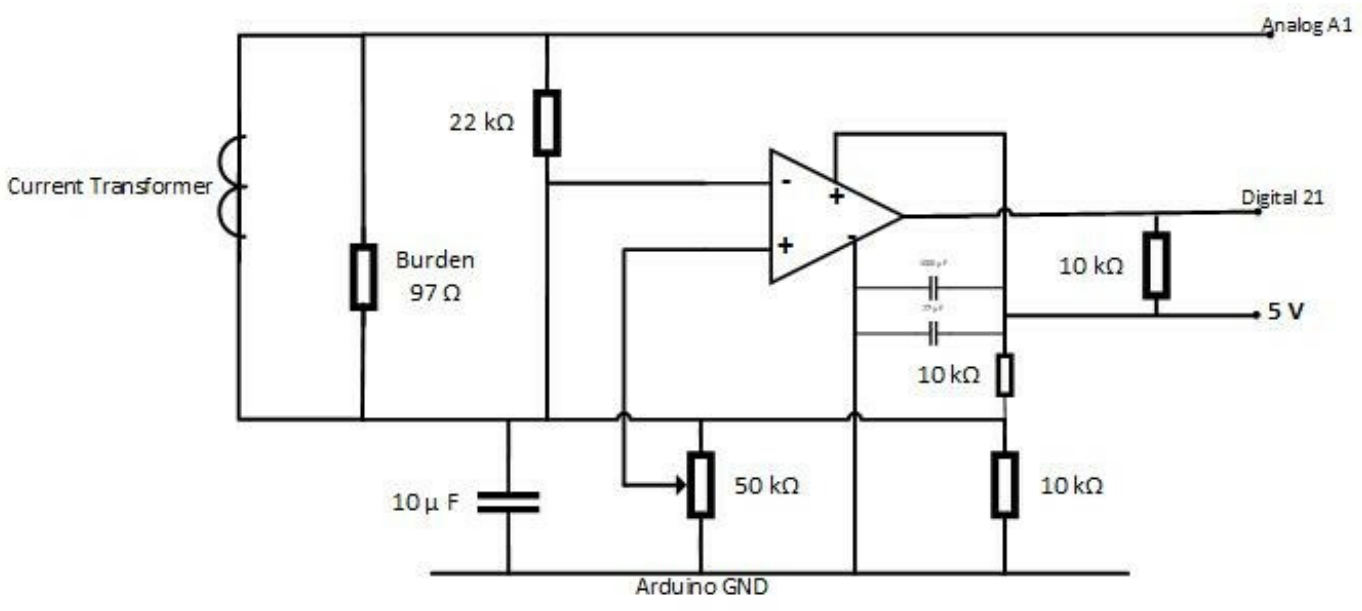

Figure 6: "Wake up" and measuring circuit scheme

After calculating the parameters, we built the prototype of the device (Fig. 7, Fig. 8).

Until we reached the final parameter values we had some obstacles to pass in the testing and validation phase. Due to the noise on the initial circuit, "waking up" was done also without connecting a consumer (Fig. 9). To reduce the noise, on the comparator feed we put 2 capacitors, one electrolytic of $1000 \mu \mathrm{F}$ (Fig. 10) and one ceramic of $27 \mu \mathrm{F}$ (Fig. 11) . Fig. 12 shows the circuit noise with both capacitors.

Another problem was that sometimes the signal was not strong enough to generate the necessary interruption. To solve this problem, we put a $10 \mathrm{kOhm}$ resistance between the output 


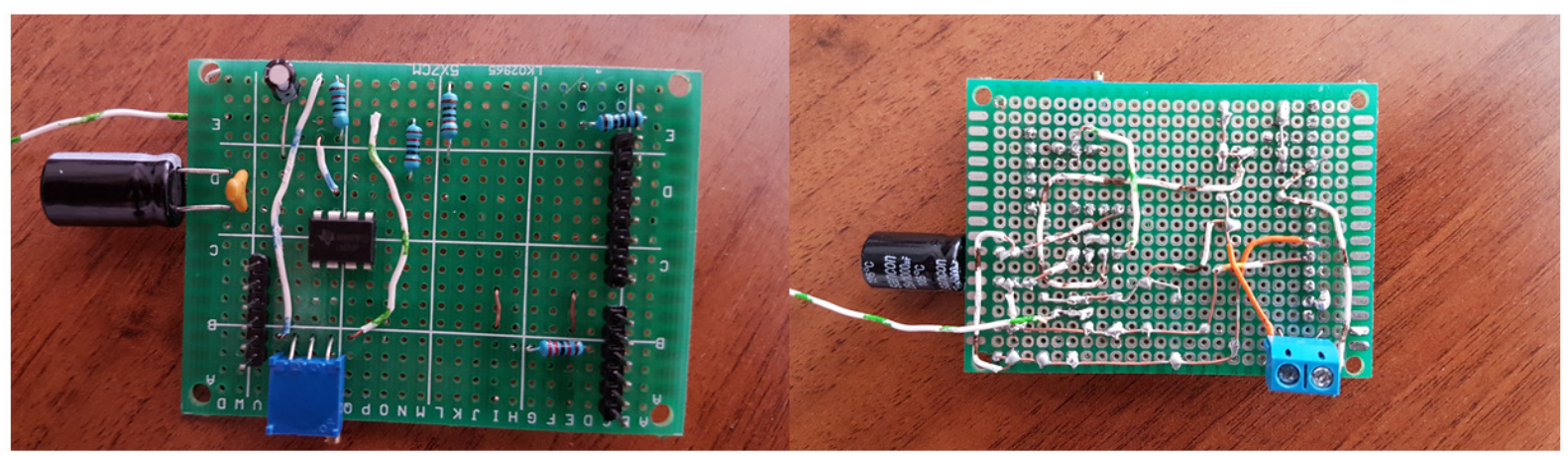

Figure 7: Circuit prototype

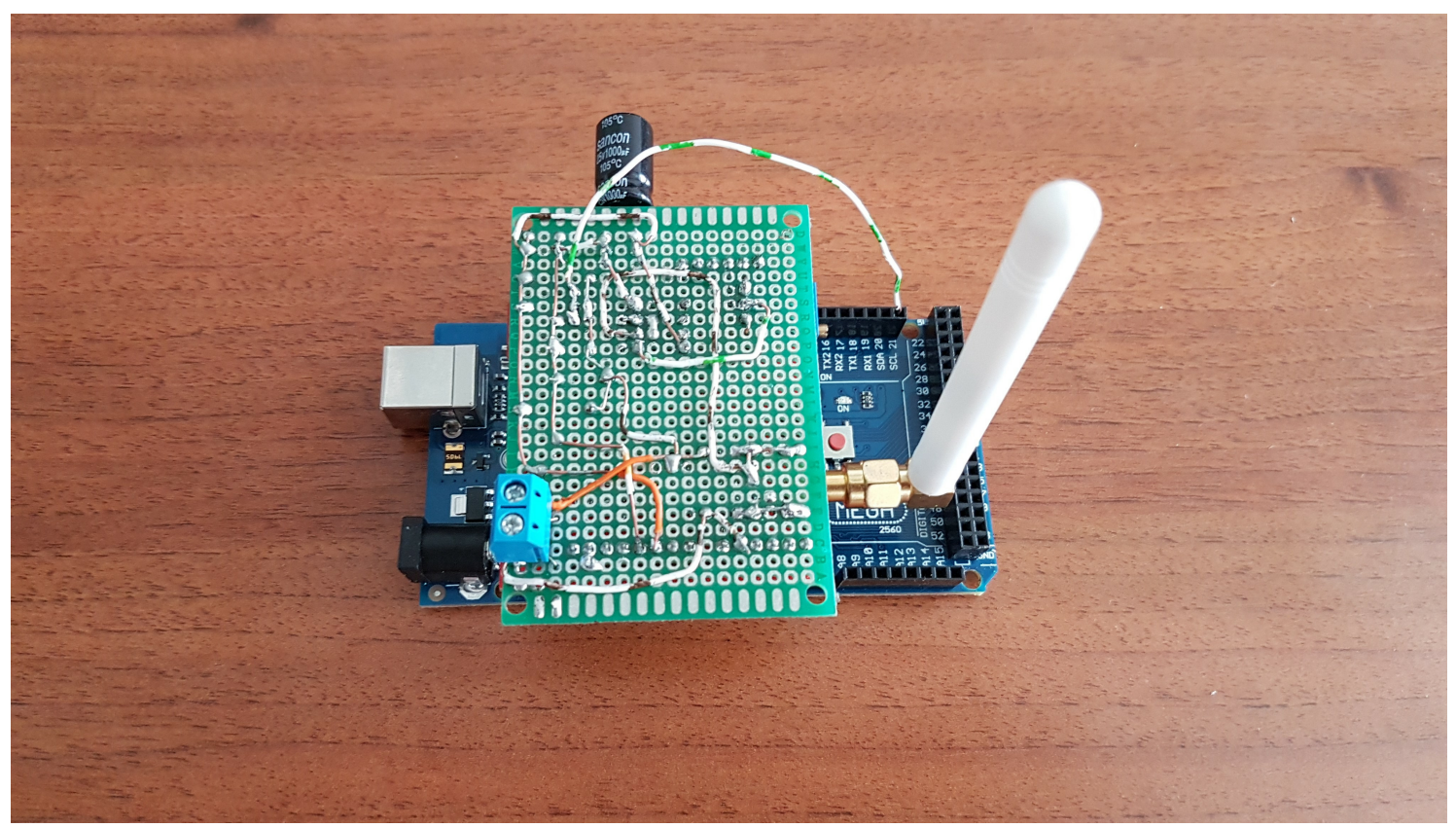

Figure 8: Device prototype

of the amplifier and the $5 \mathrm{~V}$ supply of the microcontroller. Fig. 13 shows the final shape of the circuit.

Considering these corrections, the final form of this device (Fig. 14) was mounted on production lines in several factories, collecting and sending data through LoRaWAN to a gateway behind which is an application that allows data analysis, graphs, helping the process engineers optimizing the process and the energy consumption. 


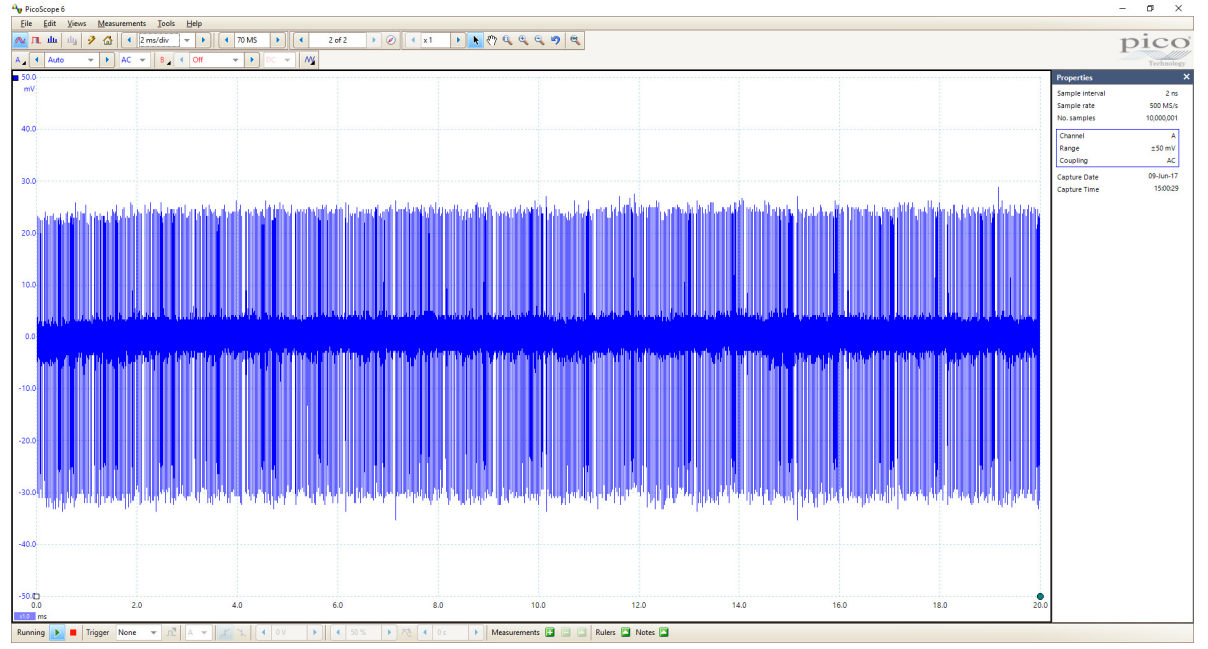

Figure 9: Circuit noise without capacitors (2 ms/div)

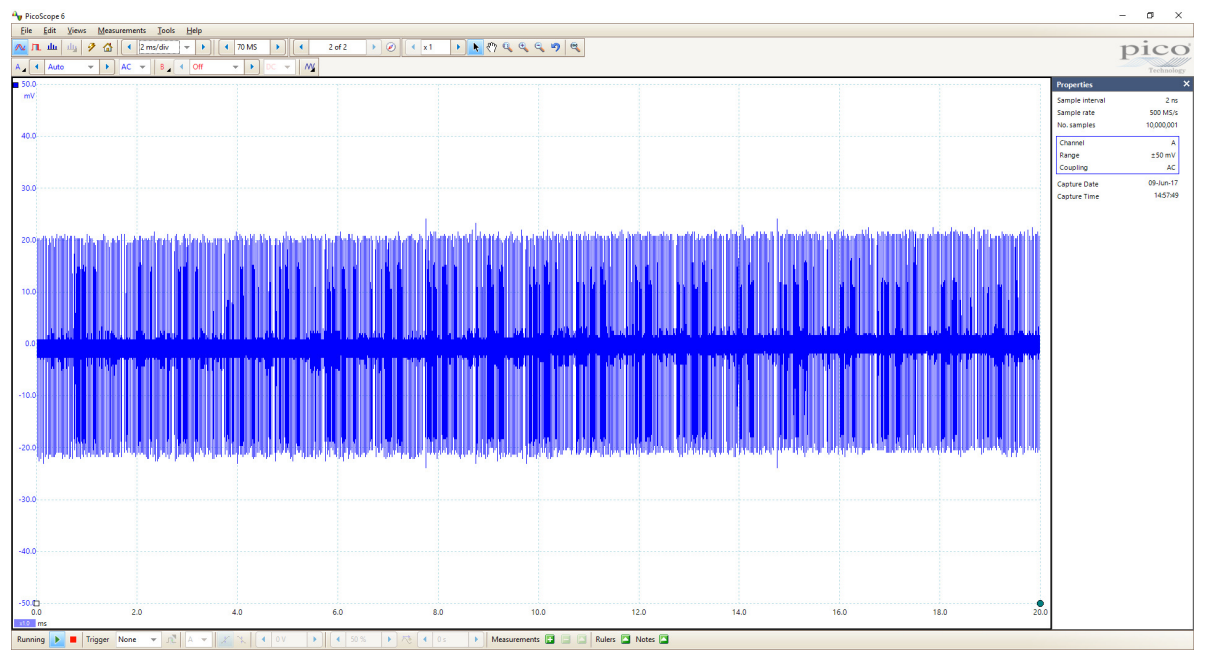

Figure 10: Circuit noise with $1000 \mu \mathrm{F}$ electrolytic capacitor (2 ms/div)

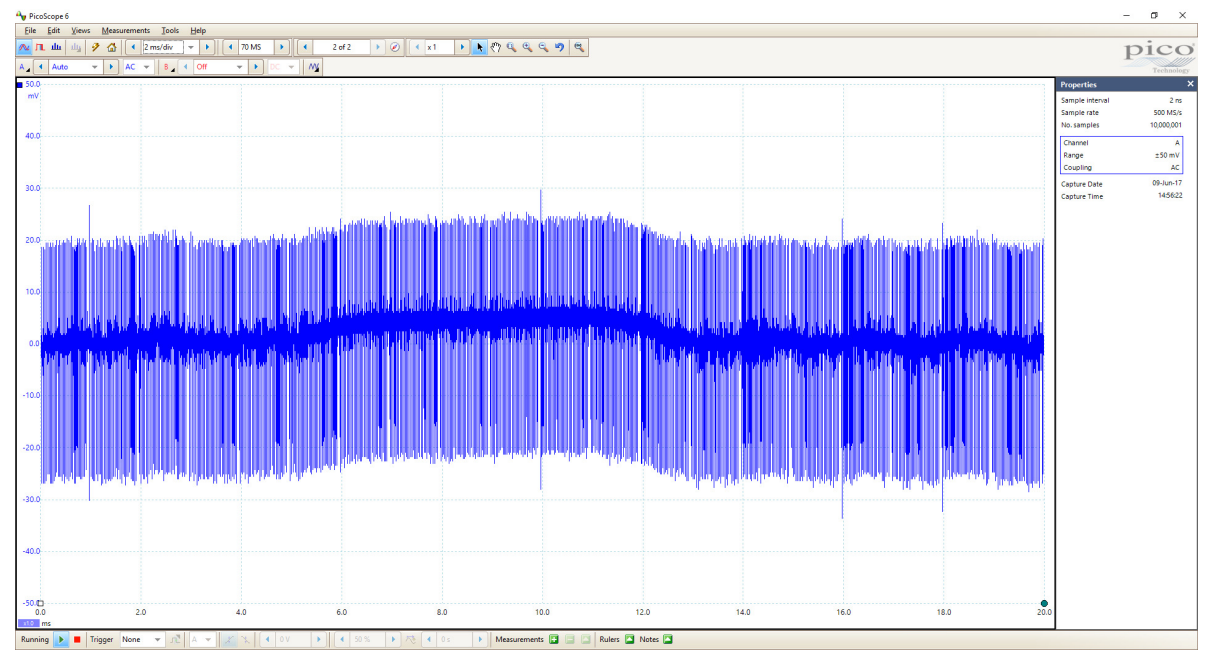

Figure 11: Circuit noise with $27 \mu \mathrm{F}$ ceramic capacitor $(2 \mathrm{~ms} /$ div $)$ 


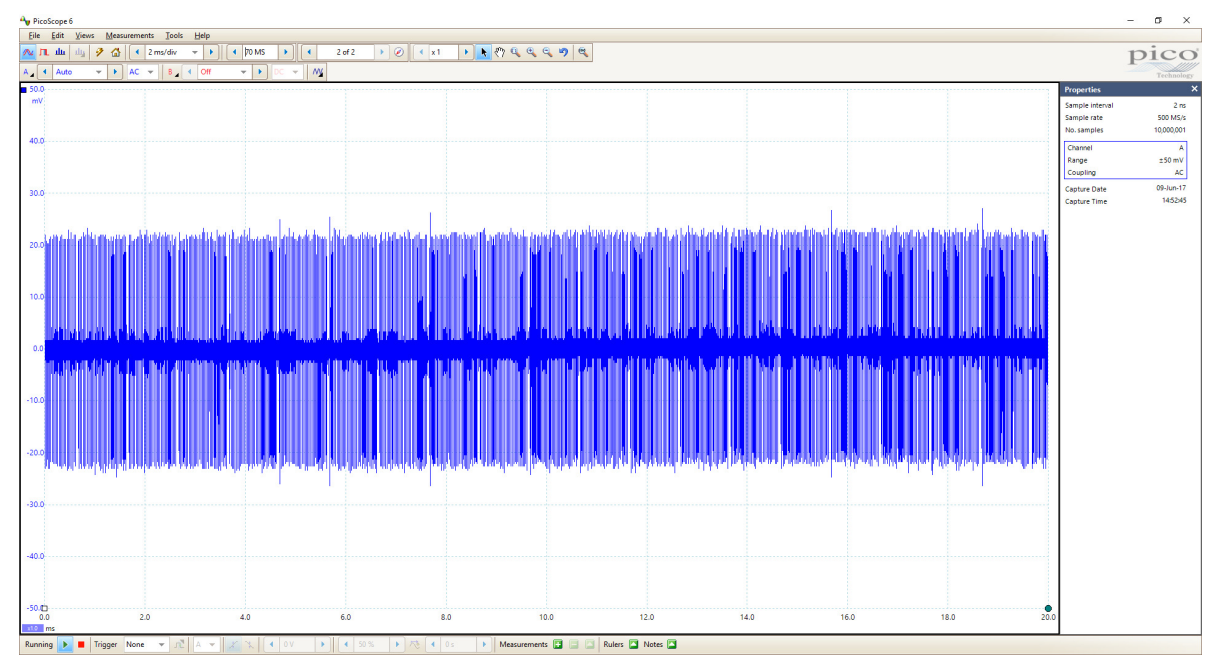

Figure 12: Circuit noise with both capacitors $(2 \mathrm{~ms} / \mathrm{div})$

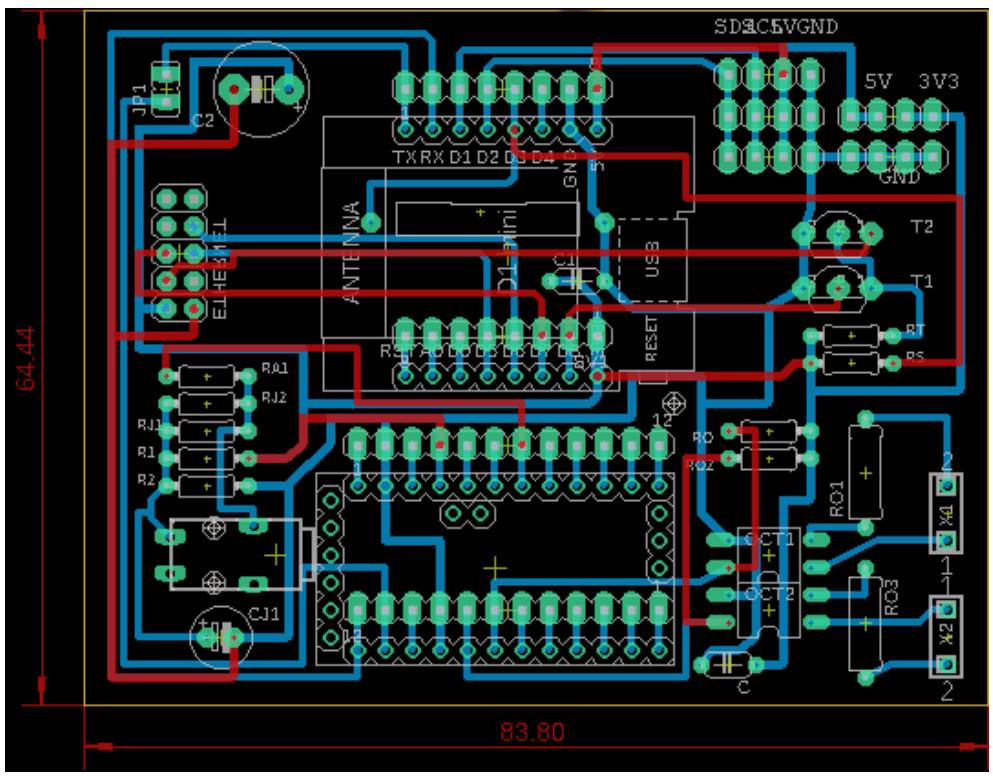

Figure 13: The final form of the circuit

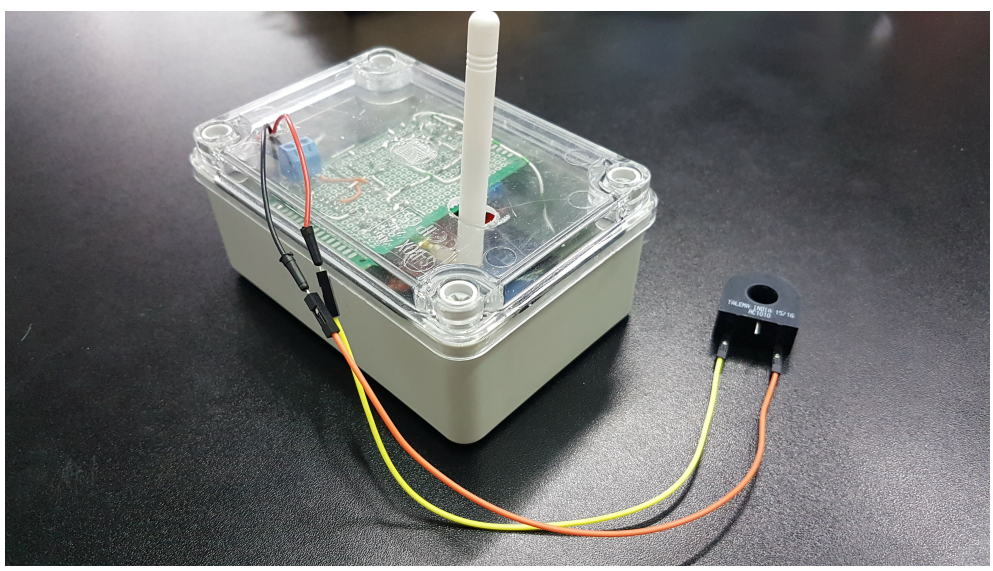

Figure 14: The final form of the device 


\section{Conclusions}

This paper addresses a topic that companies need to consider once they plan to improve the energy efficiency of their manufacturing facilities and achieve this goal thought IoT solutions. These solutions enable a very high level of awareness, being capable of being flexibly installed and collecting large quantities of energy-related data (and beyond), almost in real-time. For this reason, it is highly important to design in advance how such IoT energy monitoring solutions have to be included in the company's energy management approach.

The developed device offers a novel perspective on integrating "campus"/isolated factories (not only) in future energy management trend, using IoT solutions. Moreover, the new version of the device allows more than the previous one, such as Ethernet connection or the connection to the technological process via the programmable logic controllers, using optocouplers.

There are several situations, as is the case with these companies when more is needed, not only sensors connected via Internet; wide area coverage, low power consumption, and inexpensive wireless connectivity are necessary to achieve the goal.

The energy management practices and the proposed device offer a novel perspective on integrating energy data into manufacturing process management and related decisions at the operational level within these companies.

\section{Funding}

This work was partial supported by the Politehnica University Timisoara research grants PCD-TC-2017. The authors are addressing special thanks to the members of ETA2U Innovation.

\section{Author contributions}

The authors contributed equally to this work.

\section{Conflict of interest}

The authors declare no conflict of interest.

\section{Bibliography}

[1] Augustin, A.; Yi, J.; Clausen, T.; Townsley, W.M. (2016). A Study of LoRa: Long Range \& Low Power Networks for the Internet of Things, Journal of Sensors, 16(9), 1-18, 2016.

[2] Avotins, A.; Senfelds, A.; Apse-Apsitis P.; Podgornovs, A. (2017). IoT solution approach for energy consumption reduction in buildings: Part 1. Existing situation and problems regarding electrical consumption, Proceeding of the 58th International Scientific Conference on Power and Electrical Engineering of Riga Technical University (RTUCON), 31-36, 2017.

[3] Avotins, A.; Podgornovs, A.; Senfelds, A.; Vegeris M. (2018). IoT Solution Approach for Energy Consumption Reduction in Buildings: part 2. Measurement Setup and Practical Data Analysis, Proceedings of the17th International Scientific Conference on Engineering for Rural Developmen, 923-929, 2018.

[4] Berthelsen, E.; Morrish, J. (2015). Forecasting the internet of things revenue opportunity, Tech. Rep., 2015. 
[5] Cattani, M.; Boano, C.A.; Romer, K. (2017). An Experimental Evaluation of the Reliability of LoRa Long-Range Low-Power Wireless Communication, Journal of Sensor and Actuator Networks, 6(2), 1-18, 2017.

[6] Garetti, M.; Taisch, M. (2012). Sustainable manufacturing: trends and research challenges, Journal ofProdustion Planning \& Control, 23(2), 83-104, 2012. ]

[7] Kharel, J.; Shin, S. Y. (2017). Smart Health Monitoring System of Employee for Smart Factory, Proceedings of Symposium of the Korean Institute of communications and Information Sciences, 1-6, 2017.

[8] Nokia (2016). LTE evolution for IoT connectivity, Tech. Rep., 2016.

[9] Petajajarvi, J.; Mikhaylov, K.; Roivainen, A.; Hanninen, T.; Pettissalo M. (2015). On the coverage of LPWANs: range evaluation and channel attenuation model for lora technology, Proceedings of the 14th International Conference on ITS Telecommunications (ITST), 5559, 2015.

[10] Neumann, P.; Montavont, J.; NoĂ ̌̆l, T. (2016). Indoor Deployment of Low-Power Wide Area Networks (LPWAN): a LoRaWAN case study, Proceedings of the 2th International Conference on Wireless and Mobile Computing, Networking and Communications (WiMob), 54-58, 2016.

[11] Petjjrvi, J.; Mikhaylov, K.; Hmlinen, M.; Iinatti, J. (2016). Evaluation of LoRa LPWAN technology for remote health and wellbeing monitoring, Proceedings of the 10th International Symposium on Medical Information and Communication Technology (ISMICT), 1-5, 2016.

[12] Rizzi, M.; Ferrari, P.; Flammini, A.; Sisinni, E. (2017). Evaluation of the IoT LoRaWAN Solution for Distributed Measurement Applications, Journal of Instrumentation and Measurement, 66(12), 1-18, 2017.

[13] Raza, U.; Kulkarni, P.; Sooriyabandara M. (2017). Low Power Wide Area Networks: An Overview, Journal of Communications Surveys \& Tutorials, 19(2), 855-873, 2017.

[14] Simo, A.; Barbulescu C.; Kilyeni, S.; Dragos, C. (2018). LoRa based Energy Efficiency Improving in Manufacturing Processesn; Proceeding of the 7th International Conference on Computers Communications and Control (ICCCC), 156-161, 2018.

[15] Shrouf, F.; Miragliotta, G. (2015). Energy management based on Internet of Things: practices and framework for adoption in production management, Journal of Cleaner Production, 100, 235-246, 2015.

[16] Weinert, N.; Chiotellis, S.; Seliger G. (2011). Methodology for planning and operating energy-efficient production systems, CIRP Annals, 60(1), 41-44, 2011.

[17] Xiong, X.; Zheng, K.; Xu, R.; Xiang, W.; Chatzimisios, P. (2015). Low power wide area machine-to-machine networks: key techniques and prototype, IEEE Communications Magazine, 53(9), 64-71, 2015. 